\title{
VALIDATION OF AN HPLC-DAD-ESI/MS/MS METHOD FOR THE CLASSIFICATION OF GREEN TEAS
}

\author{
Jingbo Yu, Nengsheng Ye, Xuexin Gu ${ }^{*}, \mathrm{Ni}$ Liu \\ Department of Chemistry, Capital Normal University, Beijing, China 100048 \\ * Corresponding author, Address: Department of Chemistry, Capital Normal University, \\ No.105, Xisanhuan North Road, Beijing 100048, P. R. China, Tel:+86-10-68902490, \\ Email: guxuexin@263.net
}

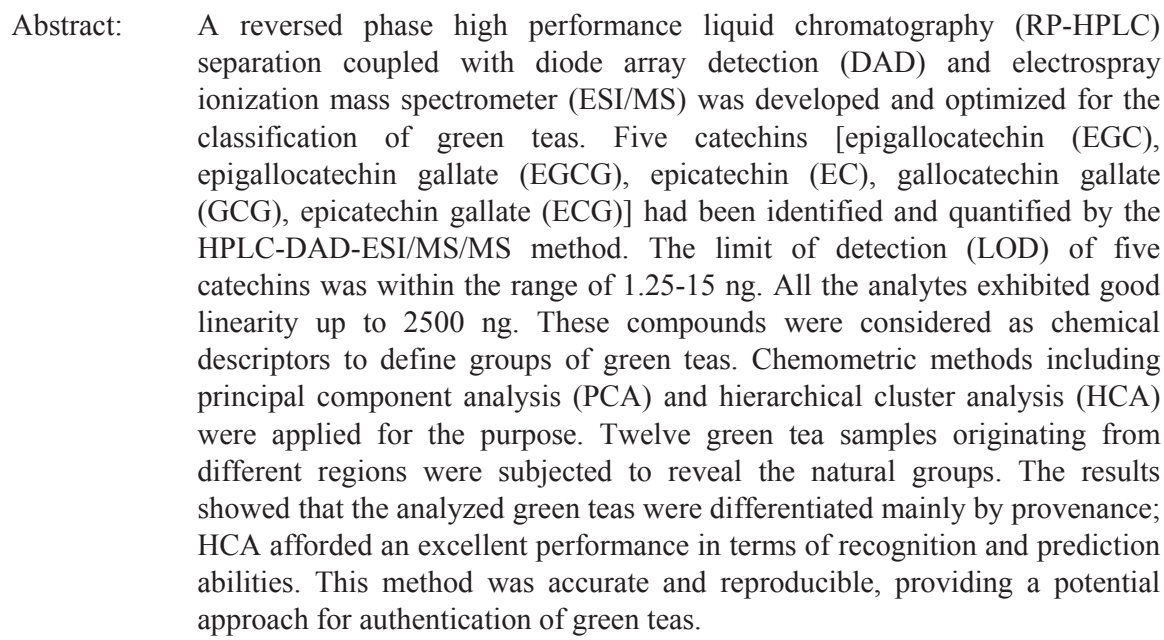

Key words: green tea, catechins, classification, HPLC

Please use the following format when citing this chapter:

Yu, J., Ye, N., Gu, X. and Liu, N., 2009, in IFIP International Federation for Information Processing, Volume 295, Computer and Computing Technologies in Agriculture II, Volume 3, eds. D. Li, Z. Chunjiang, (Boston: Springer), pp. 1707-1719. 


\section{INTRODUCTION}

Green tea is the most popular beverage in China. Recent studies have proved that green tea confers beneficial effects to human's health, especially for the effects of anti-oxidation, anti-cancer and prevention of cardiovascular disease. It is generally believed that catechins (Fig.1), the principal bioactive compounds of green tea, are responsible for the claimed therapeutic activities (Sakanaka et al., 1989; Blentine, 1997; Yang, 1997). Therefore it is essential to establish routine quality control method for the analysis of catechins in green tea.

Several analytical methods for analyzing catechins had been developed (Lee et al., 2000; Bonoli et al., 2003; Zhao et al., 2006; Shaghaghi et al., 2008; Tsukagoshi et al., 2008). Among the methods, HPLC was widely applied due to its excellent reproducibility and accuracy. Although most of HPLC methods were practicable, many established strategies were rarely available for simultaneous analysis of the content of catechins (Goto et al., 1996; Dalluge et al., 1998; Wang et al., 2000; Wang et al., 2003; Yao et al., 2004; Nishitani et al., 2004; Pelillo et al., 2004; Zhu et al., 2004; Liang et al., 2005; Owuor et al., 2007), no available method was suitable for authentication of tea. However, tea authenticity was vital to tea quality. Therefore, methods to guarantee tea authenticity, based on chemical analysis and sophisticated data analysis procedures, were demanded by consumers and tea producers. Chemometric techniques are commonly employed in order to develop system for the differentiation of geographical origin and fraud detection.

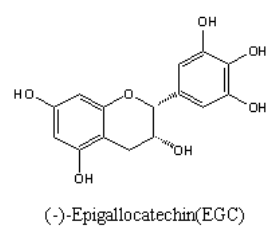

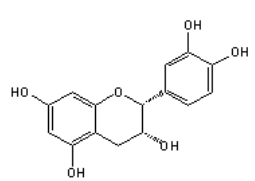

(-)-Epicatechin(EC)

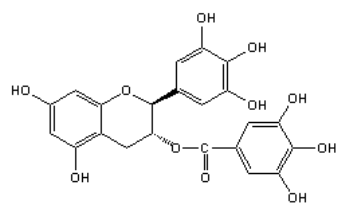

(-)-Gallocatechin gallate(GCG)
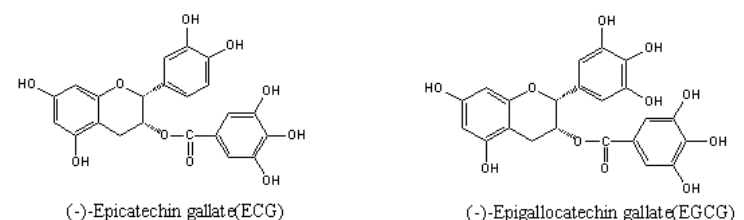

Figure 2: Chemical structures of catechins

This paper reported an application of chemometric techniques to HPLC data for the classification of green teas based on chemical characteristics. In this study, twelve green tea samples of three species originating from Henan, Jiangsu and Zhejiang province had been investigated to define groups. Five 
catechins in samples had been simultaneous analyzed and utilized as chemical descriptors to apply chemometric methods including principal component analysis (PCA) and hierarchical cluster analysis (HCA) for grouping.

\section{MATERIALS AND METHODS}

\subsection{Materials}

The standard chemicals of (-)-epigallocatechin [(-)-EGC], (-)epigallocatechin gallate [(-)-EGCG], (-)-epicatechin [(-)-EC], (-)gallocatechin gallate [(-)-GCG] and (-)-epicatechin gallate [(-)-ECG] were purchased from Sigma Chemical Co. (St. Louis, MO, USA). Methanol (HPLC grade) was purchased from Fisher Scientific (Fairlawn, NJ, USA). The water was prepared with a Millipore Milli-Q purification system (Bedford, MA, USA). Other reagents were analytical grade.

Chinese green tea samples were purchased from local tea shops (Beijing, China).

\subsection{Preparation of samples}

$1.00 \mathrm{~g}$ tea leaves were accurately weighed and extracted with $200 \mathrm{~mL}$ boiling water in $100^{\circ} \mathrm{C}$ water bath. After 30 min extraction, the extraction mixture was cooled to room temperature and filtered into a $250 \mathrm{~mL}$ volumetric flask and made to volume with water. Approximately $2 \mathrm{~mL}$ sample solution was filtered through a $0.45 \mu \mathrm{m}$ polytetrafluroethylene (PTFE) filer prior to HPLC analysis.

\subsection{Preparation of catechin standard solutions}

Stock solution, $2.5 \mathrm{mg}$ of each standard chemical was accurately weighed into a 5-mL volumetric flask, dissolved and made to volume with methanol, avoided light at $-20^{\circ} \mathrm{C}$. Working standard solutions were prepared by $2-1000$ fold dilution of the stock solutions with methanol prior to HPLC analysis.

\subsection{Analytical determinations}

An HPLC-DAD-ESI/MS/MS system comprising an Agilent 1200 series liquid chromatography system(Palo Alto, CA, USA) equipped with a 
G1322A degasser, a G1311A quaternary pump, a G1329A autosampler, a G1316A thermostat column compartment, a G1315B diode array detector and a Micromass Q-TOF mass spectrometer (Manchester, UK) was used. The column was a C18 reversed phase Agilent Zorbax SB-C18 $5 \mu \mathrm{m}(250 \times$ $4.6 \mathrm{~mm})$ with a Zorbax SB-C18 $5 \mu \mathrm{m}(30 \times 4.6 \mathrm{~mm})$ guard column. Mobile phases consisted of $0.5 \%$ acetic acid in water $(\mathrm{v} / \mathrm{v})$ (eluent $\mathrm{A})$ and methanol (eluent B). The gradient elution system was: $0-5 \mathrm{~min}, 13 \% \mathrm{~B} ; 5-20 \mathrm{~min}$, linear gradient from 13 to $20 \% \mathrm{~B} ; 20-25 \mathrm{~min}, 20 \% \mathrm{~B} ; 25-35 \mathrm{~min}$, linear gradient from 20 to $25 \% \mathrm{~B} ; 35-40 \mathrm{~min}, 25 \% \mathrm{~B} ; 40-50 \mathrm{~min}$, linear gradient from 25 to $40 \%$ B; $50-60 \mathrm{~min}$, linear gradient from 40 to $80 \%$ B. Elution was performed at a solvent flow rate of $0.8 \mathrm{~mL} / \mathrm{min}$. Detection was accomplished with a diode array detector and chromatograms were recorded at $278 \mathrm{~nm}$. The column was maintained at $35^{\circ} \mathrm{C}$. The injection volume was $5 \mu \mathrm{L}$.

Mass spectra of catechins were operated in negative mode using an electrospray ionizing source with nitrogen as desolvation gas. Spray chamber parameters: ion spray voltage, $3.5 \mathrm{kV}$; desolvation gas temperature, $300{ }^{\circ} \mathrm{C}$; desolvation gas flow, $400 \mathrm{~L} / \mathrm{h}$. The full-scan mass was acquired over the range $50-1500 \mathrm{~m} / \mathrm{z}$. Cone voltage was $10 \mathrm{~V}$ in full-scan mode, and the voltage for selected ion monitoring (SIM) was set at $30 \mathrm{~V}$.

\subsection{Data analysis}

\subsubsection{Principal component analysis}

Principal component analysis (PCA) is a statistical tool commonly used for classification of data. PCA can reduce the dimensionality of the data considerably, enabling effective visualization regression and classification of multivariate data (Qian et al., 1994). PCA compresses a large number of variables to a much smaller number of principal components (PCs) that capture the majority of variance in the data by means of mathematical transformation. The PCs can be displayed in a scatter diagram, presenting the individual samples as points in a lower-dimensional (generally 2-D or 3D) space.

\subsubsection{Hierarchical cluster analysis}

Hierarchical cluster analysis (HCA) is performed to classify samples on the basis of the similarities of their measured properties. Objects are grouped in clusters in terms of their nearness in the multidimensional space. The elements or clusters are joined with the criterion that the sum of heterogeneities of all clusters shall increase as little as possible. In this work, 
the distance matrix was calculated using Euclidean distances. From the distance matrices the dendograms were created using the Ward algorithm.

\section{RESULTS AND DISCUSSIONS}

\subsection{Optimization of HPLC-DAD-ESI/MS/MS conditions}

\subsubsection{Column temperature}

Column temperature was critical for the separation of catechins. The effect of column temperature on catechins was investigated in the range of $20 \sim 45^{\circ} \mathrm{C}$ (Fig.2). The experimental results indicated that with the increase of column temperature, the retention times of analytes decreased.

At lower column temperature, the chromatograms showed baseline merging of analytes and other components. Baseline separation could be obtained upon $30^{\circ} \mathrm{C}$. As could be seen from Figure 2, the difference of capacity factor ( $\left.\mathrm{k}^{\prime}\right)$ between adjacent analytes reached a maximum at $35^{\circ} \mathrm{C}$. Hence, column temperature of $35^{\circ} \mathrm{C}$ was chosen for further studies.

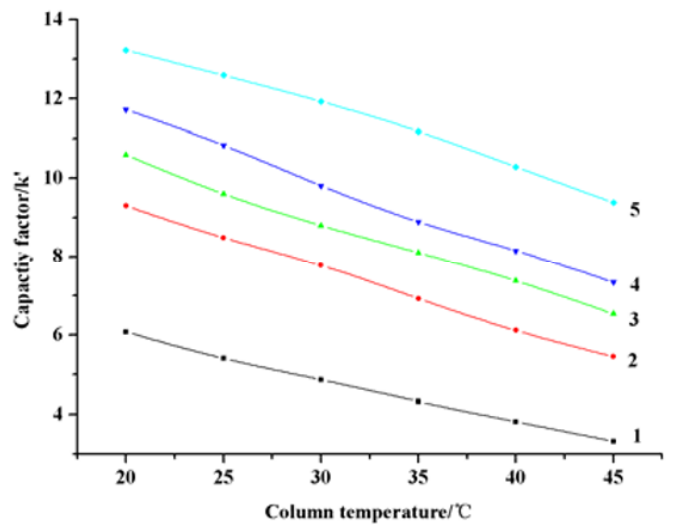

Figure 2: Effect of column temperature on capacity factor on Zorbax-SB C18 column; 1.EGC 2.EGCG 3.EC 4.GCG 5.ECG

\subsubsection{Acidity of mobile phase}

Acidity of the mobile phase was also essential for the separation and peak shape of catechins (Dalluge et al., 1998). In this paper, acetic acid was utilized as modifier of the mobile phase. To optimize the acidity of mobile phase, the analysis was done at different volume fraction of acetic acid in 
eluent A viz. $0,0.25 \%, 0.5 \%, 0.75 \%, 1.0 \%, 1.5 \%$ (Fig. 3 ). It was found that with increasing the acidity of mobile phase, the retention time of catechins decreased and peak shape became sharper.

The complete separation and well-resolved, symmetrical, sharp peaks could be obtained at $0.5 \%$, above which the separation effect had no obvious change. However, higher acidity of mobile phase could have negative impact on the function of stationary phase. Therefore, the volume fraction of acetic acid in eluent $\mathrm{A}$ was fixed at $0.5 \%$.

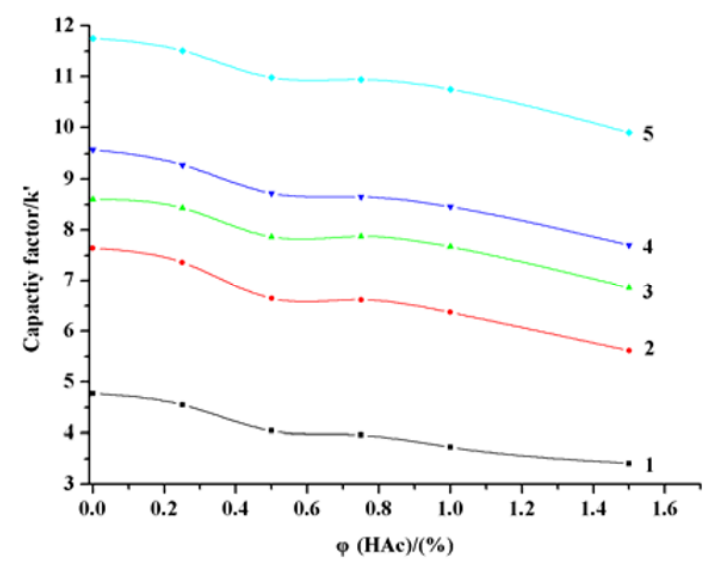

Figure 3: Effect of acidity of mobile phase on capacity factor on Zorbax-SB C18 column; 1.EGC 2.EGCG 3.EC 4.GCG 5.ECG

\subsubsection{Detected wavelength}

Wavelengths between 210 and $220 \mathrm{~nm}$ or between 270 and $280 \mathrm{~nm}$ were ordinarily applied to the detection of catechins (Dalluge et al., 1998; Goto et al., 1996). Catechins showed maximum absorbance at $210 \mathrm{~nm}$. However, the baseline shifted seriously at $210 \mathrm{~nm}$ due to the change of the mobile phase. As the baseline was smooth during the gradient elution at $278 \mathrm{~nm}$, the chromatograms were recorded at $278 \mathrm{~nm}$.

\subsection{Optimization of sample preparation conditions}

\subsubsection{Extraction temperature}

Extraction temperature could obviously affect the extraction efficiency due to the increase in solubility of catechins in water with temperature. In this work, the effect of extraction temperature on the extraction efficiency over the temperatures interval of $50^{\circ} \mathrm{C}$ to $100^{\circ} \mathrm{C}$ with an extraction time of 30 min was studied (Fig.4). It was demonstrated that the extraction efficiency 
for all target analytes except EGCG increased with temperature and maximum extraction efficiency was obtained at $100^{\circ} \mathrm{C}$. The most significant effect was observed between $90^{\circ} \mathrm{C}$ and $100^{\circ} \mathrm{C}$. To obtain better extraction efficiency, $100^{\circ} \mathrm{C}$ was selected as extraction temperature for subsequent experiment.

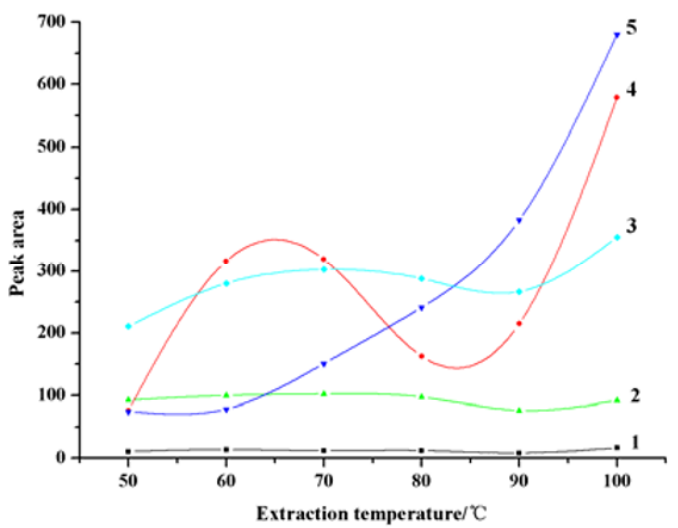

Figure 4: Effect of extraction temperature on extraction efficiency; 1.EGC 2.EC 3.ECG 4.EGCG 5.GCG

\subsubsection{Extraction time}

Extraction time was another factor that influenced the extraction efficiency. The effect of extraction time on the extraction efficiency was investigated in the range of 10 120 min (Fig.5). As could be seen from Fig.5, the peak area for each analyte increased sharply within $30 \mathrm{~min}$. It appeared that the time effect faded after $60 \mathrm{~min}$, which might be caused by oxidation of catechins. Therefore, 30 min was most suitable for extraction.

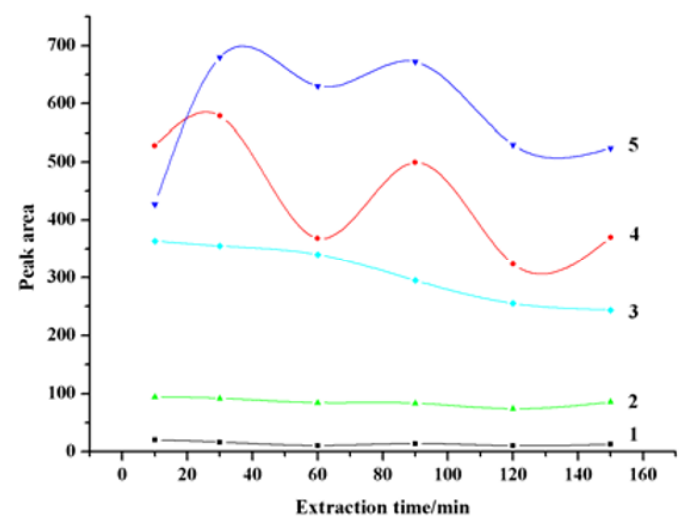

Figure 5: Effect of extraction time on extraction efficiency; 1.EGC 2.EC 3.ECG 4.EGCG 5.GCG 


\subsection{Method validation}

\subsubsection{Linearity and limit of detection}

Calibration curves of the catechins were constructed using six levels of concentration which covered the concentration ranges expected in samples (Fig.6). The characteristics of calibration curve, including the range of linearity, the square of correlation coefficient $\left(\mathrm{R}^{2}\right)$ and limit of detection (LOD) was listed in Table 1. The LOD was evaluated as a signal equals three times of noise $(\mathrm{S} / \mathrm{N}=3)$. It could be seen that an excellent linearity was observed for each analyte in the range studied.

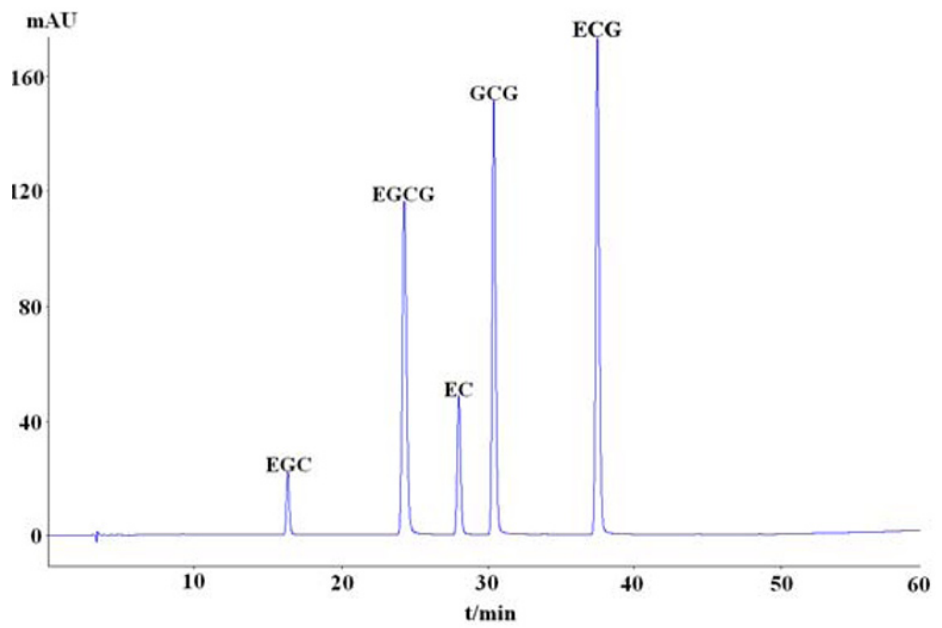

Figure 6: Chromatogram of catechin standards monitored at $278 \mathrm{~nm}$.

Table 1: Characteristics of the calibration curve

\begin{tabular}{llccc}
\hline Compound & Calibration curve & $\begin{array}{c}\text { Correlation coefficient } \\
\left(r^{2}\right)\end{array}$ & $\begin{array}{c}\text { Liner range } \\
(n g)\end{array}$ & $\begin{array}{c}\text { LOD } \\
(n g)\end{array}$ \\
\hline EGC & $\mathrm{Y}=0.5828 \mathrm{x}+11.062$ & 0.9986 & $50 \sim 2500$ & 15 \\
EGCG & $\mathrm{Y}=4.5871 \mathrm{x}+83.4$ & 0.9978 & $50 \sim 2500$ & 0.75 \\
EC & $\mathrm{Y}=1.496 \mathrm{x}+28.661$ & 0.9982 & $50 \sim 2500$ & 5 \\
$\mathrm{GCG}$ & $\mathrm{Y}=4.721 \mathrm{x}+82.72$ & 0.9983 & $50 \sim 2500$ & 0.75 \\
ECG & $\mathrm{Y}=5.7258 \mathrm{x}+112.42$ & 0.9977 & $50 \sim 2500$ & 1.5 \\
\hline
\end{tabular}

\subsubsection{Precision}

To test the precision of the assay method, one of the samples to be analysed was injected five times under the HPLC conditions described above. The relative standard deviations (RSD) of relative peak area for all analytes were within $1.15 \%$. 


\subsubsection{Stability}

The stability of the catechins in the sample solution was determined by analyzing the same sample after 2, 4, 6, 12, 24, 30, $48 \mathrm{~h}$ of preparation. The experiment showed stability within 24 hours when the sample was kept at room temperature.

\subsubsection{Reproducibility}

The reproducibility was evaluated by the RSD of relative peak area of five analytes in various batches of samples. It was found that the maximum RSD was less than $4.20 \%$.

\subsection{Identification of catechins by HPLC-DAD-ESI- MS/MS}

Catechins were identified by comparing retention time, UV spectra, $\mathrm{m} / \mathrm{z}$ of their quasi-molecular ions, and $\mathrm{MS}^{2}$ fragmentation patterns of unknown peaks to the standards. The typical HPLC chromatogram and total ion current chromatogram of Biluochun, a well-known green tea, was shown in (Fig.7 and Fig.8), while Table 2 showed the retention times, MS and $\mathrm{MS}^{2}$ data and the identification results for the peaks numbered in the chromatogram.

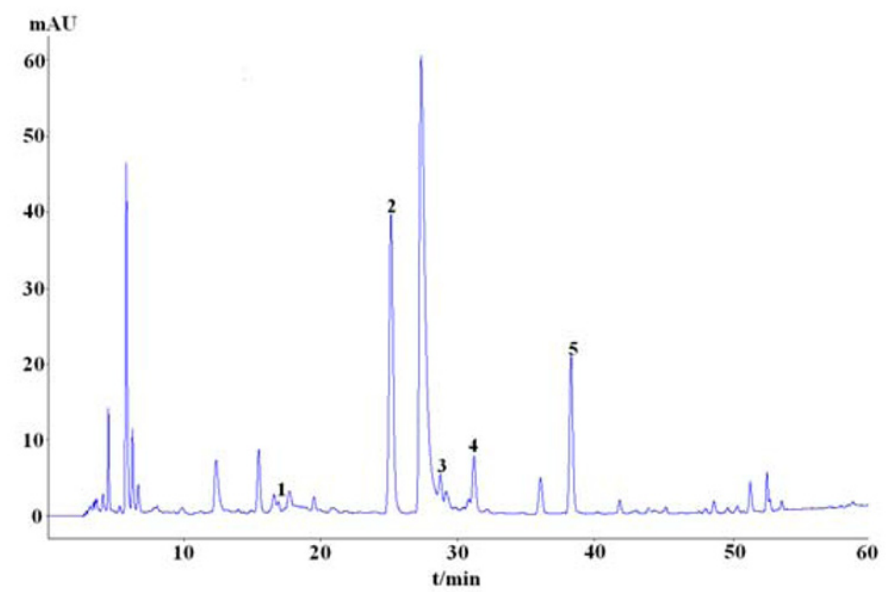

Figure 7: Chromatogram of Biluochun monitored at $278 \mathrm{~nm}$. Peak identification: 


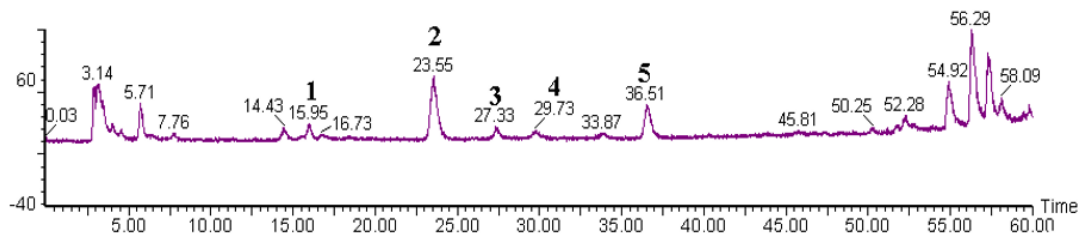

Figure 8: Total ion current chromatogram of Biluochun; Peak identification:

1.EGC 2.EGCG 3.EC 4.GCG 5. ECG

Table 2: Retention time, molecular weight and the $\mathrm{m} / \mathrm{z}$ values of selective ions for identification of catechins

\begin{tabular}{cccccl}
\hline No & $R t$ & Compound & Molecularweight & Selectiveion $(\mathrm{m} / \mathrm{z})$ & \multicolumn{1}{c}{$\mathrm{MS}^{2}(\mathrm{~m} / \mathrm{z})$} \\
\hline 1 & 16.7 & EGC & 306 & 305 & $219,167,137,125$ \\
2 & 25.0 & EGCG & 458 & 457 & $305,169,125$ \\
3 & 28.5 & EC & 290 & 289 & $203,151,109$ \\
4 & 31.0 & GCG & 458 & 457 & $305,169,125$ \\
5 & 38.2 & ECG & 441 & 441 & $289,245,169,125$ \\
\hline
\end{tabular}

\subsection{Analysis of green tea samples}

Twelve Chinese green tea samples of three species (Xinyang maojian, Biluochun, Xihu longing) were analyzed for catechins by employing the developed method. The content of five analytes were calculated as milligram per gram of dry weight (Tab.3)

Table 3: Content of five catechins for 12 tea samples ( $\mathrm{mg} / \mathrm{g}$ of $\mathrm{dw})$

\begin{tabular}{ccccccccc}
\hline No & Sample $^{*}$ & Grade & Source & $E G C$ & $E G C G$ & $E C$ & $G C G$ & $E C G$ \\
\hline 1 & MJ & 4 & Hennan & 1.3 & 12.3 & 12.6 & 19.7 & 4.0 \\
2 & MJ & 3 & Hennan & 4.6 & 21.3 & 9.3 & 29.0 & 6.6 \\
3 & MJ & 2 & Hennan & 1.2 & 17.7 & 8.4 & 25.9 & 7.0 \\
4 & MJ & 1 & Hennan & 1.5 & 15.6 & 7.7 & 25.0 & 6.6 \\
5 & BLC & 4 & Jiangsu & 4.3 & 24.1 & 8.7 & 27.2 & 11.9 \\
6 & BLC & 3 & Jiangsu & 1.2 & 15.2 & 7.7 & 20.2 & 9.8 \\
7 & BLC & 2 & Jiangsu & 1.4 & 23.1 & 6.9 & 25.1 & 10.6 \\
8 & BLC & 1 & Jiangsu & 2.1 & 19.7 & 7.2 & 20.3 & 9.5 \\
9 & LJ & 4 & Zhejiang & 1.5 & 24.2 & 10.8 & 14.6 & 6.0 \\
10 & LJ & 3 & Zhejiang & 1.8 & 7.5 & 10.2 & 10.5 & 3.5 \\
11 & LJ & 2 & Zhejiang & 1.9 & 6.8 & 10.9 & 11.4 & 2.4 \\
12 & LJ & 1 & Zhejiang & 1.9 & 12.2 & 12.5 & 12.7 & 3.5 \\
\hline
\end{tabular}

* MJ: Xinyang Maojin; BLC: Biluochun; LJ: Xihu Longjin

\subsection{Hierarchical cluster analysis}

HCA applied the information obtained from the measured variables to reveal the natural clusters exiting between the studied samples. The 
Euclidean distance was used as similarity measurement and the Ward's method was utilized as amalgamation rule to obtain the hierarchical associations. The data matrix used for the multivariate analysis was composed of a $12 \times 5$ data matrix ( 12 samples and 5 variables). The result of HCA was presented as a dendogram (Fig.9). The resulting dendogram was interpreted to classify the twelve Chinese green tea samples in three groups based on the similarity of the five studied parameters. Group A was composed of 4 Xinyang maojian. Group B comprised 4 Biluochun. Group C contained 4 Xihu longjing. This meant that $100 \%$ correct classification was achieved by HCA.

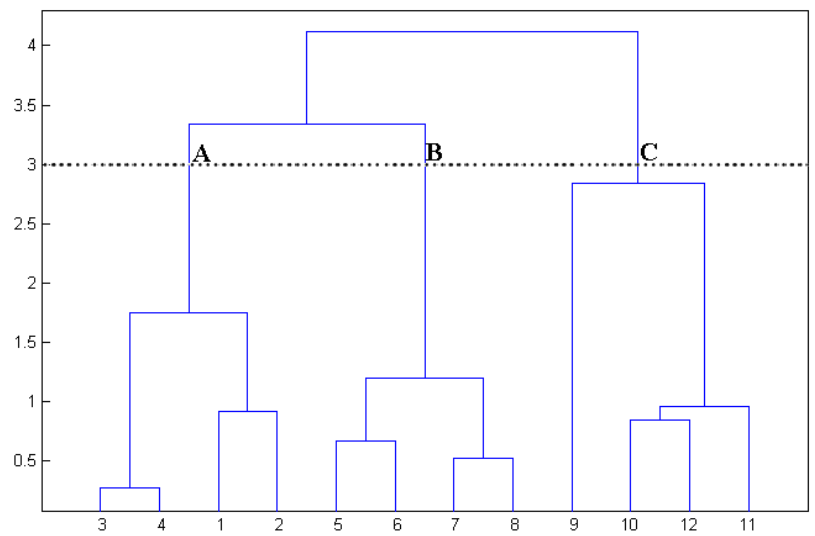

Figure 9: Hierarchical cluster analysis dendgram of 12 green tea samples using Euclidean distance; 1-4 Xinyang maojian, 5-8 Biluochun, 9-12 Xihu longjing

\subsection{Principal component analysis}

PCA technique was applied to reduce the number of dimensions present in the data matrix (reducing 5 variables to 2 PCs in this study), to select the most discriminating parameters, and to investigate the overall variation of data. Rotation of principal components was carried out using the Varimax normalized method and Kaiser Criterion. Varimax normalized procedure for eigenvector rotation resulted in two principal components (PC1 and PC2), which explained $85 \%$ of the total variance. Figure 10 showed the result of the PCA analysis of the 12 green tea samples. The first principal component (PC1) contained $43.9 \%$ of the total variance and the second component (PC2) represented $41.1 \%$ of the total variance. The twelve green tea samples with various species and origins were grouped into three clusters. Representation of the green tea employing PCA was effective and reasonable as the first two components accounted for $85 \%$ of the total variance. 


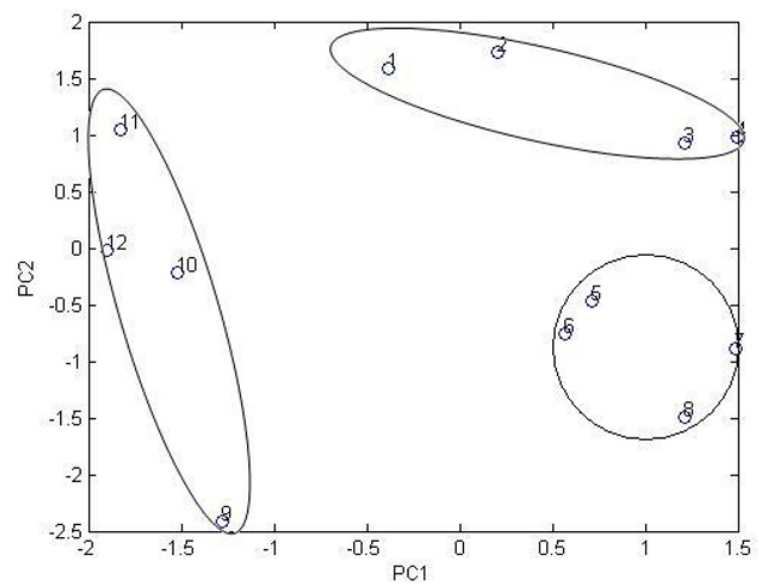

Figure 10: Samples in the space defined by first and second principal components; 1-4 Xinyang maojian, 5-8 Biluochun, 9-12 Xihu longjing

\section{CONCLUSIONS}

In this study, an HPLC-DAD-ESI/MS/MS method was developed and optimized for analyzing green teas. Five catechins had been simultaneously identified and quantified. The HPLC tandem with DAD and ESI-MS/MS provided multi-dimension qualitative information comparing with ordinary HPLC analysis of catechins.

Chemometric methods (PCA and HCA) had proved to be available approaches to the classification of green teas based on the content of catechins. Twelve Chinese green tea samples originating from three regions were subjected to reveal the natural grouping among the source. PCA provided information on the overall components of green teas and the overlap of the clusters. HCA rendered three significant green tea groups on the basis of similarities in their chemical properties with percentage of correct classification of $100 \%$. Variations in the chemical compositions of green teas were mostly caused by environments (climate, geologic formation).

The result suggested that PCA and HCA were available for the classification of green teas. This study confirmed a potential of HPLC in connection with chemometric method for authentication of green tea.

\section{REFERENCES}

Balentine D. Tea and health. Critical Reviews in Food Science and Nutrition, 1997, 37: 691692 
Bonoli M, Pelillo M, Toschi TG, Lercker G. Analysis of green tea catechins: comparative study between HPLC and HPCE. Food Chemistry, 2003, 81: 631-638

Dalluge JJ, Nelson BC, Thomas JB, Sander LC. Selection of column and gradient elution system for the separation of catechins in green tea using high performance liquid chromatography. Journal of Chromatography A, 1998, 793: 265-274.

Goto T, Yoshida Y, Kiso M, Nagashima H. Simultaneous analysis of individual catechins and caffeine in green tea. Journal of Chromatography A, 1996, 749: 295 - 299.

Lee BL, Ong CN. Comparative analysis of tea catechins and theaflavins by high-performance liquid chromatography and capillary electrophoresis. Journal of Chromatography A, 2000, 881: 439-47.

Liang YR, Zhang LY, Lu JL. A study on chemical estimation of pu-erh tea quality. Journal of the Science of Food and Agriculture, 2005, 85: 381-390

Nishitani E, Sagesaka YM. Simultaneous determination of catechins, caffeine and other phenolic compounds in tea using new HPLC method. Jornal of Food Composition and Analysis, 2004, 17: 675-685

Owuor PO, Obanda M. The use of green tea (Camellia sinensis) leaf flacan-3-ol composition in predicting plain black tea quality potential. Food Chemistry, 2007, 100: 873-844

Pelillo M, Bonoli B, Biguzzi B, Bendini A, Toschi TG, Lercker G. An investigation in the use of HPLC with UV and MS-electrospray detection for quantification of tea catechins. Food Chemisty, 2004, 87: 465-470

Qian G, Gabor G, Gupta RP. Principal components selection by the criterion of the minimum mean difference of complexity. Journal of Multivariate Analysis, 1994, 49: 55 - 75

Sakanaka S, Kim M, Taniguchi M, Yamamoto T. Antibacterial substance in Japanese green tea extract against Streptococus mutans, a carcinogenic bacterium. Agriculture Biology and Chemistry, 1989, 53: 2307-2311

Shaghaghi M, Manzoori JL, Jouyban A. Determination of total phenols in tea infusions, tomato and apple juice by terbium sensitized fluorescence method as an alternative approach to the Folin - Ciocalteu spectrophotometric method. Food Chemistry, 2008, 108: 695-701

Tsukagoshi K, Taniguichi, Nakajima R. Analysis of antioxidants using a capillary electrophoresis with chemiluminescence detection system. Analytica Chimica Acta, 2007, 589: 66-70.

Wang H, Helliwell K, You X. Isocratic elution system for the determination of catechins, caffeine and gallic acid in green tea using HPLC. Food Chemistry, 2000, 68: 115 - 121.

Wang HF, Provan GJ, Helliwell K. HPLC determination of catechins in tea leaves and tea extracts using relative response factors. Food chemistry, 2003, 81: 307-312

Yang CS. Inhibition of carcinogenesis by tea. Nature, 1997, 389: 134-135

Yao LH, Jiang YM, Datta N , Singanusong R, Liu X, Duan J, Raymonte K, Lisle A, Xu Y. HPLC analyses of avanols and phenolic acids in the fresh young shoots of tea (Camellia sinensis) grown in Australia. Food Chemistry, 2004, 84: 253-263

Zhao JW, Chen QS, Huang XY, Fang CH. Qualitative identification of tea categories by near infrared spectroscopy and support vector machine. Journal of Pharmaceutical and Biomedical Analysis, 2006, 41: 1198-1204

Zhu XL, Chen B, Ma M, Luo XB, Zhang F, Yao SZ, Wan ZT, Yang DJ, Hang HW. Simultaneous analysis of theanine, chlorogenic acid, purine alkaloids and catechins in tea samples with the help of multi-dimension information of on-line high performance liquid chromatography/electrospray-mass spectrometry. Journal of Pharmaceutical and Biomedical Analysis, 2004, 34: 695-704 